\title{
Xi Jin, China CDC's Chief Expert of Maternal and Child Health
}

\author{
Peter $\mathrm{Hao}^{1, \alpha} ; \mathrm{Xi} \mathrm{Jin}^{2, \alpha}$; Xiaoqi Wang ${ }^{1}$; Zhenjun $\mathrm{Li}^{1}$; Jingjing $\mathrm{Xi}^{1, *,}$; Feng $\operatorname{Tan}^{1, *}$
}

Editorial Our new column Profiles is a new series that aims to introduce China CDC's chief experts, major decisionmakers, and key contributors that ensure the high standard of operation of China CDC. These articles are meant to present high-achieving individuals of China CDC and will describe the background, achievements, and paths to success of the eponymous individuals.

To start, Profiles will first cover individuals from China CDC's Chief Expert System. To strengthen the establishment of a team of public health talent, China CDC expanded the career development capacity for its most well-known experts under Director-General George F. Gao's directive: "To further perfect the system of chief experts and continuously expand the influence of chief experts". In December 2019, China CDC selected the nominations of chief experts based on the needs of key areas of public health and the academic level and professional influence of the experts. After domestic peer review and comprehensive evaluation by China CDC's academic committee, 13 individuals (in no particular order) were appointed to lead 13 major public health fields: 1) Guizhen Wu, Chief Expert of Biosafety; 2) Yiming Shao, Chief Expert of AIDS; 3) Weiping Wu, Chief Expert of Parasitic Diseases; 4) Huaqing Wang, Chief Expert of Immunization Planning; 5) Zunyou Wu, Chief Expert of Epidemiology; 6) Linhong Wang, Chief Expert of Chronic Diseases; 7) Xu Su, Chief Expert of Radiation Protection; 8) Wenhua Zhao, Chief Expert of Nutrition; 9) Tao Li, Chief Expert of Occupational Health; 10) Liubo Zhang, Chief Expert of Disinfection; 11) Jiaqi Ma, Chief Expert of Health Informatics; 12) Xi Jin, Chief Expert of Maternal and Child Health; and 13) Qiyong Liu, Chief Expert of Vector Biology.

In response to the prevention and control of the coronavirus disease 2019 (COVID-19) pandemic, the chief experts were dispatched to the front lines and highly-affected areas to supervise the response, tasked with leading scientific research to shape the response and the preparation of technical documents for prevention and control, and were responsible for promptly supporting government decisionmaking and responding to social concerns as the scientific authorities. The voices of these chief experts has played an important role in the effective management of the epidemic in their respective professional fields.

China CDC plans to dynamically adjust the fields represented by the chief experts based on the country's major public health needs by enriching the chief expert's system and guiding the chief experts to play the role of "public opinion leaders" in academic communication and health communication at home and abroad.

Our first publication in Profiles, therefore, is China CDC's Chief Expert of Maternal and Child Health, Jin $\mathrm{Xi}$, who has over 30 years of experience in developing and managing maternal and child healthcare $(\mathrm{MCH})$ institutions. With the advent of the $31^{\text {st }}$ World Population Day on July 11, 2020, the public health community needs to prioritize $\mathrm{MCH}$ as a fundamental basis for the improvement of health globally.

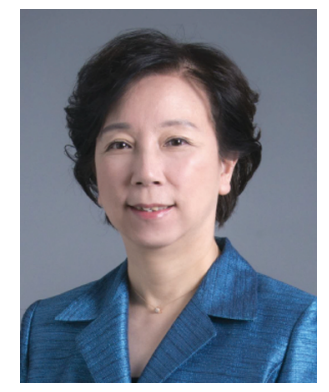

Before beginning her long and fruitful career with China CDC, Xi Jin was working in the Beijing Municipal Child Health Institute. Fifteen years of work had given her a deeper understanding of China's maternal and child health network system and the foundation of individual clinical services. She won the United Arab Emirates Health Foundation Prize from the World Health Organization in her capacity as a representative of maternal and child health in China, in which she had been working fifteen years as the Deputy Director of the National Center for Women and Children's Health (NCWCH), China CDC. As one of the founders, Jin's expertise in maternal and child healthcare (MCH) allowed her to help establish national MCH institutions and start the process of building and developing China's robust MCH systems. 
China has the largest population of women and children in the world, which poses huge demands and challenges for $\mathrm{MCH}$. Jin frequently reorients her strategies on answering the question: "How do we meet this huge demand of women and their children in China?" She believes that a complete $\mathrm{MCH}$ system and institution is the most fundamental step for providing high quality services to meet the population's needs. Therefore, with keen technical anticipation and clarity of thought, Jin led her team to establish and perform a series of studies on the departments of $\mathrm{MCH}$ institutions including human resources allocation standards, construction standards, equipment configuration standards, bed settings in obstetric departments, and service item cost accounting, which were then utilized to build construction standards for $\mathrm{MCH}$ institutions. Based on these studies, her team has developed 27 management documents and over 20 clinical guidelines, which covered almost all aspects of MCH services. Jin also introduced advanced concepts of international medical quality and safety management in China and compiled a series of books on $\mathrm{MCH}$ quality and safety management. These accomplishments mentioned above were all pioneering initiatives in the world. As the first prize winner of the government-ministry-level $\mathrm{MCH}$ award, Jin has made substantial contributions to accelerate the standardized construction and management process of $\mathrm{MCH}$ institutions and of the $\mathrm{MCH}$ service network in China.

Based on the improved Chinese $\mathrm{MCH}$ system, many important $\mathrm{MCH}$ programs including the prevention of mother-to-child transmission of HIV, syphilis, and hepatitis B (PMTCT) program, have made remarkable achievements in the world. As the team leader, Jin has been deeply committed to the PMTCT program and led her team to actively commit to the elimination of mother-to-child transmission in China. She was responsible for cooperative projects between China's National Health Commission (NHC) and the United Nations International Children's Fund (UNICEF) on PMTCT and special research projects on HIV/AIDS-related issues funded by China's Ministry of Science and Technology. These works provided comprehensive technical support for China's action to eliminate mother-to-child transmission of HIV/AIDS, syphilis, and hepatitis B. Therefore, in 2016, she was invited as the only national-level observer to participate in the WHO's accreditation for the elimination of mother-to-child transmission of HIV/AIDS in Thailand.

As one of the think tank experts of the Women and Children's Working Committee in the State Council of China and a member of national technical groups of various national $\mathrm{MCH}$ programs, Jin is extensively involved in the development of national $\mathrm{MCH}$ policies and also stays open-minded and actively looking for opportunities on the introduction, localization, training, and implementation of new MCH technologies. She pays attention to research on key, difficult, and current issues in $\mathrm{MCH}$ in order to promote management capacity. The many areas of $\mathrm{MCH}$ covered by this research include emergency obstetric care, neonatal intensive care, neonatal asphyxia resuscitation, early essential newborn care (EENC), newborn healthcare, early childhood development, adolescent health and development, sexual and reproductive health, health education, and health promotion. The research of these subjects has significantly promoted the comprehensive development of maternal and child health care service in China.

Jin loves her job very much. "The $\mathrm{MCH}$ work is very meaningful," she always says. "It's something that matters for everyone, including individuals and families. I enjoy this job which brings them health and happiness, and I feel very lucky for having the chance to contribute my lifetime to a career that I love and can always be passionate about." To now, Jin has been engaged in maternal and child health work for more than 30 years and dedicated to $\mathrm{MCH}$ for her entire career life with a persistent work attitude and a strong sense of professionalism and responsibility. She has gained respect and recognition worldwide. In view of her outstanding contributions and great achievements, she has won the award of National Advanced Individual for the Protection of Women and Children's Rights, the Development Contribution Award for Public Health and Preventive Medicine, the 2018 Influential Figures of Health Communication, and many other awards for her scientific and technological achievements.

As a part of the field of public health, $\mathrm{MCH}$ plays an important role in promoting the health of women and children, and, as a member of the China $\mathrm{CDC}$, NCWCH has played an important role in ensuring the quality and availability of $\mathrm{MCH}$. Now and in the future, Jin and her colleagues will continue to commit to the development and progress of China's $\mathrm{MCH}$ and to progress towards the goals of Healthy China 2030 Plan and the United Nations Sustainable Development Goals.

doi: $10.46234 /$ ccdcw2020.144

\# Corresponding authors: JingjingXi, xijj@chinacdc.cn; Feng Tan, tanfeng@chinacdc.cn.

\footnotetext{
Chinese Center for Disease Control and Prevention, Beijing, China; ${ }^{2}$ National Center for Women and Children's Health, Chinese Center for Disease Control and Prevention, Beijing, China.

\& Joint first authors.
}

Submitted: May 10, 2020; Accepted: July 06, 2020 